\title{
Combined spatial and Kalman filter estimation of optimal soil hydraulic properties
}

\author{
Anthony T. Cahill, ${ }^{1}$ Fabrizio Ungaro, ${ }^{2}$ Marc B. Parlange, ${ }^{3}$ Michael Mata, ${ }^{4}$ and \\ Donald R. Nielsen ${ }^{4}$
}

\begin{abstract}
A method for determining optimal parameters for a field-scale hydraulic conductivity function is presented and tested on soil moisture and matric potential data measured at several locations in a field drainage experiment. The change in moisture content over time at the individual locations is modeled using Richards' equation, and an optimization for the hydraulic conductivity parameters is performed using a merit function derived from the Kalman filter, which allows consideration of measurement and process noise. The spatial correlation among the different measurement points is explicitly taken into account using the covariance between points in the calculation of the process noise covariance matrix. It is shown that the standard deviation of the effective hydraulic conductivity function estimated by the Kalman filter method applied to all measurements is significantly less than the standard deviations estimated by simple averaging of the parameters derived using other methods applied to the individual point moisture time series.
\end{abstract}

\section{Introduction}

The description of hydrological processes is often complicated by the disparity of the scales at which measurements are made and the scale over which the processes of interest operate. For the movement of water through the unsaturated zone, this problem is acute, since measurements of unsaturated hydraulic conductivity are generally done for a specific point in the field and can vary significantly over the larger scale of interest [Nielsen et al., 1973].

The specific upscaling problem addressed herein considers a large-scale hydraulic conductivity function (or a set of parameters for such a function) derived from measurements obtained on a small scale. Much of the work which has been done on estimating large-scale hydraulic conductivities has focused on developing models relating the small-scale observations to the large-scale parameters. Drawing on extensive work on the similar problem for the saturated zone, researchers investigating the unsaturated zone have applied stochastic approaches to the problem [Yeh et al., 1985a, b; Mantoglou and Gelhar, 1987a, b, c; Mantoglou, 1992]. In this approach local hydraulic conductivity is represented as the sum of a large-scale hydraulic conductivity value and a small-scale local fluctuation, which is a stochastic field. By using spectral perturbation techniques, an expression for the effective conductivity can be derived in which the effective conductivity is a function of the large-scale conductivity and the correlations among various small-scale variables. Another approach that treats the hydraulic conduc-

\footnotetext{
${ }^{1}$ Department of Civil and Environmental Engineering, Texas A\&M University, College Station.

${ }^{2}$ Soil Genesis and Ecology Institute, CNR-IGES, Florence, Italy.

${ }^{3}$ Department of Geography and Environmental Engineering, Johns Hopkins University, Baltimore, Maryland.

${ }^{4}$ Department of Land, Air and Water Resources, University of California, Davis.

Copyright 1999 by the American Geophysical Union.
}

Paper number 1998WR900121.

0043-1397/99/1998WR900121\$09.00 tivity as a random field is that taken by Chen et al. [1994], in which a spatially averaged Richards' equation is derived that also includes a mean (large-scale) conductivity and conductivity correlations as controlling parameters. A different approach for deriving large-scale hydraulic conductivity utilizing volume averaging of an unknown deterministic conductivity field has been developed by Quintard and Whitaker [1988].

The necessary parameters for the spatially based models described above can be calculated from information from large-scale unsaturated zone investigations such as the Las Cruces experiment [Wierenga et al., 1991]. In practice, however, it can be easier to acquire a time series of soil moisture content for a smaller number of points than it is to measure hydraulic conductivity at a greater number of locations. In addition, for certain hydrologic processes, such as drainage or infiltration, the physics are reasonably well understood, so that a model for the inverse problem is clearly available. For this reason, past work has often focused on the analysis of time series taken under these conditions for the determination of unsaturated hydraulic conductivity [e.g., Libardi et al., 1980]. These methods ultimately provide estimators of the parameters in a hydraulic conductivity equation by means of linear regression of a moisture content dependent term against a time-dependent term. These methods are based on data from a single point, however, and as will be seen, they can give quite variable results depending on the measurement location.

In this paper we propose a method of deriving optimal parameters for an effective large-scale hydraulic conductivity that concurrently considers both the temporal and spatial variations of the moisture content. Optimal parameters are defined as those hydraulic conductivity parameters that minimize the mean square error of the moisture content time series, based on all the imperfect measurements of soil moisture content from a number of spatial points. The resulting parameters will be those that best describe the movement of water through the soil when all the different locations observed are considered. This definition of optimal is the one put forth in the development of the Kalman filter, and we will use the extended 


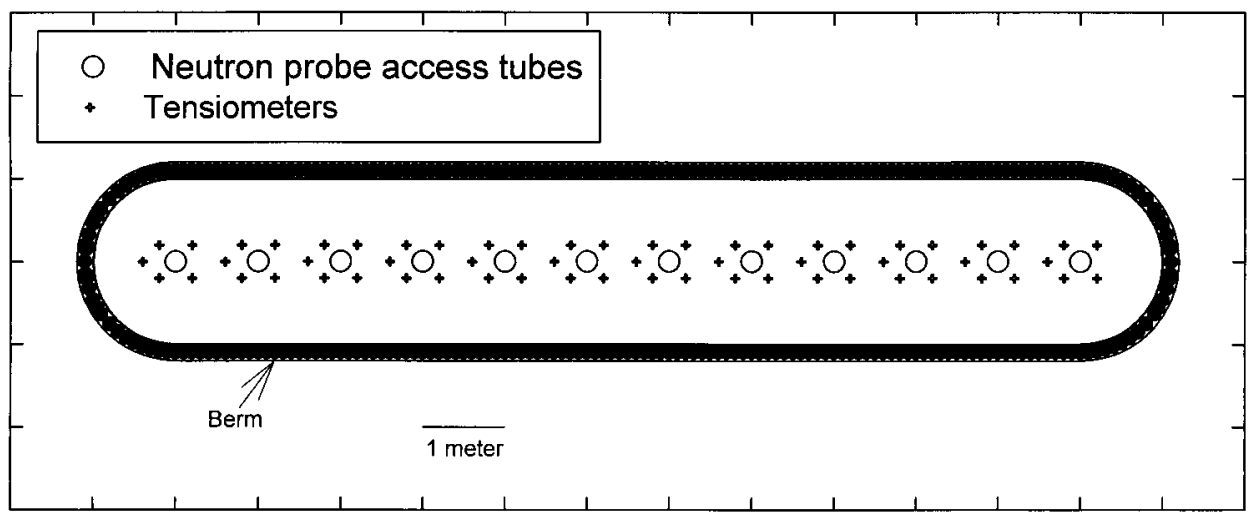

Figure 1. Plan view of the experimental transect. The area inside the berm was first ponded for 10 days, and then the bare soil was covered with black plastic and soil. Note that the neutron probe access tubes and tensiometers are not shown to scale. They are enlarged for easier viewing, but their positions are correct.

Kalman filter to estimate this mean square error, given a set of parameters. Certain parameters in the Kalman filter will have to be estimated, and geostatistical methods will be applied to estimate them, using the relationship between semivariograms and spatial covariance. By taking into account the spatial correlation among the measurement points, this technique extends an earlier method which yielded unsaturated hydraulic conductivity for a single point [Katul et al., 1993].

We investigate the estimation of a field-scale hydraulic conductivity from a set of soil moisture measurements taken by a neutron probe during drainage after ponding. This experiment was chosen to explore the use of the extended Kalman filter with spatially correlated noise because of its ease of execution and so that the results could be compared to classic methods of determining hydraulic conductivity from a drainage time series.

\section{Experiment}

A transect of neutron probe access tubes was installed on the University of California's Campbell Research Tract, in Davis, California. The soil at the Campbell Tract is Yolo clay loam and has been found in previous studies to have a correlation length of $1 \mathrm{~m}$ [Parlange et al., 1993]. The tubes were placed $1 \mathrm{~m}$ apart in an east-west direction and were deep enough to allow neutron probe moisture measurements to be taken at depths up to $80 \mathrm{~cm}$. Readings were taken at depths of 10, 20,35, 50, and $65 \mathrm{~m}$. Surrounding each access tube at approximately 10 $\mathrm{cm}$ distance was a ring of five tensiometers, which provided matric potentials at depths roughly between the moisture measurements. Figure 1 shows a plan view of the transect.

The transect was ponded to a depth of $10 \mathrm{~cm}$ for 10 days, until a steady state infiltration condition was established. The water was then removed, and the surface of the plot was covered with plastic to prevent evaporation. A thin layer of soil was also placed on top of the plastic to insulate the moist ground somewhat from diurnal temperature fluctuations. Soil moisture and matric potential readings were taken three times a day for the first 17 days of drainage and then once a day for the subsequent 15 days.

For the purposes of the analysis in this paper the volumetric soil moisture readings were multiplied by the depth over which they were presumed to represent and then summed to yield a total amount of water (in centimeters) for the column depth of $65 \mathrm{~cm}$. The hydraulic gradient was then derived by finding the linear slope between the matric potentials from the third and fourth deepest tensiometers, which bracketed the bottom of the column depth of $65 \mathrm{~cm}$.

\section{Theory}

\subsection{Time Series Modeling: Kalman Filtering}

The applicability of Kalman filtering to estimate optimal coefficients in a hydraulic conductivity function has already been presented for the case of a single location in the field [Katul et al., 1993; Wendroth et al., 1993]. In this section we summarize the Kalman filter algorithm applied to a set of measurement locations, in which the resulting equations are vectorial, rather than scalar, in nature. We will describe the means through which the Kalman filter estimates an optimal time series of soil moisture, given parameters for the hydraulic conductivity function, and how it determines which parameters are best.

The equation describing the evolution of the moisture content of the soil through time is

$$
\frac{\partial \theta}{\partial t}=K(\theta) \frac{\partial h}{\partial z}
$$

where $K$ is the hydraulic conductivity; $h$ is the matric potential; $z$ is the vertical direction, positive downwards; $t$ is time; and $\theta$ is the moisture content. This equation is nonlinear, since both $K$ and $h$ are in general nonlinear functions of $\theta$. The extended Kalman filter is one method of approximating nonlinear processes, using successive linearization over time [Maybeck, 1982; Gelb, 1974]. An exact approach for the nonlinear case would involve propagating and updating the probability density function for the true moisture content, conditioned on the imperfect observations, but this is not possible in general [Maybeck, 1982]. In the Kalman filter framework it is assumed that there are two sources of noise that make the exact state of the system (the true value of the moisture content at any moment) unknowable. One source of error is from measurement uncertainty, and the other source arises from a random forcing of the system in time; that is, the change in the system from one time to another is not uniquely determined but instead has 
some random component. For our set of measurements the relationship between the measured soil moisture content and the true soil moisture content can be written as

$$
\boldsymbol{\theta}_{t}=\mathbf{H} \mathbf{x}_{t}+\mathbf{v}_{t}
$$

where $\boldsymbol{\theta}_{t}$ is a vector consisting of the measured moisture contents

$$
\boldsymbol{\theta}_{t}=\left[\begin{array}{c}
\theta_{1, t} \\
\theta_{2, t} \\
\vdots \\
\theta_{n, t}
\end{array}\right]
$$

where $\theta_{1, t}, \theta_{2, t}, \cdots, \theta_{n, t}$ are the soil moisture measurements at spatial locations 1 through $n$ at time $t$. The vector $\mathbf{x}_{t}$ is the set of the true moisture contents at the individual stations, while $\mathbf{v}_{t}$ is a vector of white noise measurement errors with covariance matrix $\mathbf{R}_{t}$. The matrix $\mathbf{H}$ is the matrix that relates the state variables to the measurements, which in our case is the identity matrix. The change in time of an individual true moisture content $x_{i, t}$ can be described by the integral of (1) plus its individual unknown white noise forcing $w_{i, t}$ :

$$
x_{i, t+1}=x_{i, t}+\int_{t}^{t+1} K\left(x_{i, t}\right) \frac{\partial h_{i, t}}{\partial z} d \hat{t}+w_{i, t}
$$

This equation, which describes the evolution of the soil moisture through time, is also called the time propagation equation. The model white noise vector $\mathbf{w}_{t}$ has covariance matrix $\mathbf{Q}_{t}$. Throughout the paper the subscript $i$ indicates individual spatial locations (and different entries in the vector $\mathbf{x}, \partial \mathbf{h} / \partial z, \mathbf{w}_{t}$, etc.), while the $t$ indicates different times. For computational purposes it is necessary to assume a form for the $K\left(x_{i, t}\right)$ relationship; in this study we use an exponential function such that

$$
x_{i, t+1}=x_{i, t}+\int_{t}^{t+1}-A \exp \left(B x_{i, t}\right) \frac{\partial h_{i, t}}{\partial z} d \hat{t}+w_{i, t}
$$

Note that there are no subscripts on the hydraulic conductivity parameters $A$ and $B$; they are taken to be constant in time and space.

Since $\mathbf{w}_{t}$ is not known, our knowledge of the true moisture content is limited to an estimate $\hat{\mathbf{x}}_{t}$, which is the expected value of $\mathbf{x}_{t}$ conditioned on the observations. The evolution of this variable through time is simply the deterministic part of (5); since $\mathbf{w}_{t}$ has zero mean, it does not appear in the time-change equation for $\hat{\mathbf{x}}_{t}$. This estimate will differ from the true value $\mathbf{x}_{t}$ by some amount $\mathbf{e}_{t}=\mathbf{x}_{t}-\hat{\mathbf{x}}_{t}$, and the error covariance matrix $\mathbf{P}$ is defined as $E\left(\mathbf{e}_{t} \mathbf{e}_{t}^{T}\right)$, where $E$ is the expectation operator and $T$ indicates the transpose. The $\mathbf{P}$ matrix measures the variation of our estimate from the true unknown value, and the Kalman filter algorithm minimizes this error in a mean square sense.

Although we will not present the actual derivation of the other equations in the extended Kalman filter algorithm [Brown and Hwang, 1997; Maybeck, 1982; Gelb, 1974], we now describe the method.

Step 1. We begin with initial values of $\hat{\mathbf{x}}_{0}^{+}$and $\mathbf{P}_{0}^{+}\left(\equiv E\left[\left(\mathbf{x}_{0}\right.\right.\right.$ $\left.\left.\left.-\hat{\mathbf{x}}_{0}^{+}\right)\left(\mathbf{x}_{0}-\hat{\mathbf{x}}_{0}^{+}\right)^{T}\right]\right)$ where the superscript plus indicates that all measurements up to that time have been used to derive the estimates. We can project ahead and get values for $\hat{\mathbf{x}}_{1}^{-}$and $\mathbf{P}_{1}^{-}$ using the equations

$$
\hat{x}_{i, t+1}^{-}=\hat{x}_{i, t}^{+}+\int_{t}^{t+1}-A \exp \left(B \hat{x}_{i, t}^{+}\right) \frac{\partial h_{i, t}}{\partial z} d \hat{t}
$$

and

$$
\mathbf{P}_{t+1}^{-}=\mathbf{P}_{t}^{+}+\int_{t}^{t+1}\left[\mathbf{F}\left[\hat{\mathbf{x}}_{t}^{+}\right] \mathbf{P}_{t}^{+}+\mathbf{P}_{t}^{+} \mathbf{F}^{T}\left[\hat{\mathbf{x}}_{t}^{+}\right]+\mathbf{Q}_{t}\right] d \hat{t}
$$

where the superscript minus indicates that only measurements up to the previous time have been used to derive the estimate (i.e., only the measurement at $t=0$ has been used to estimate the value of $\hat{\mathbf{x}}_{1}^{-}$, and $\left.\mathbf{P}_{1}^{-} \equiv E\left[\left(\mathbf{x}_{1}-\hat{\mathbf{x}}_{1}^{-}\right)\left(\mathbf{x}_{1}-\hat{\mathbf{x}}_{1}^{-}\right)^{T}\right]\right)$. In (7), the matrix $\mathbf{F}[\hat{\mathbf{x}}]$ is given by

$$
F_{i j}=\frac{\partial\left(-A \exp \left(B \hat{x}_{i, t}^{+}\right) \frac{\partial h_{i, t}}{\partial z}\right)}{\partial x_{j, t}}
$$

Values for $A$ and $B$ have been assumed to have been given.

Step 2. Compute the Kalman gain, which determines how much to weight the projected value $\hat{\mathbf{x}}_{t}^{-}$and the measurement $\boldsymbol{\theta}_{t}$ in determining $\hat{\mathbf{x}}_{1}^{+}$. The Kalman gain is given by

$$
\mathbf{K}_{t}=\mathbf{P}_{t}^{-} \mathbf{H}^{T}\left[\mathbf{H} \mathbf{P}_{t}^{-} \mathbf{H}^{T}+\mathbf{R}_{t}\right]^{-1}
$$

where $\mathbf{R}_{t}$ is the covariance matrix of the measurement noise $\mathbf{v}_{t}$.

Step 3. The measurements are assimilated, weighted by the Kalman gain:

$$
\hat{\mathbf{x}}_{t}^{+}=\hat{\mathbf{x}}_{t}^{-}+\mathbf{K}_{t}\left[\boldsymbol{\theta}_{t}-\mathbf{H} \hat{\mathbf{x}}_{t}^{-}\right]
$$

The error covariance matrix that takes into account the measurement at time $t$ is given by

$$
\mathbf{P}_{t}^{+}=\left(\mathbf{I}-\mathbf{K}_{t} \mathbf{H}\right) \mathbf{P}_{t}^{-}\left(\mathbf{I}-\mathbf{K}_{t} \mathbf{H}\right)^{T}+\mathbf{K}_{t} \mathbf{R} \mathbf{K}_{t}^{T} .
$$

Step 4. Begin loop again by projecting ahead. Continue loop until all measurement have been used, and a time series of $\hat{\mathbf{x}}_{t}^{+}$is calculated.

Before the Kalman filter can be used to derive an optimal estimated time series of soil moisture content, certain quantities need to be specified: $\mathbf{P}_{0}^{+}, \mathbf{R}_{t}, A, B$, and $\mathbf{Q}_{t}$. The initial error covariance matrix $\mathbf{P}_{0}^{+}$needs to be specified; however, it was found that the choice of any reasonable value of $\mathbf{P}_{0}^{+}$made little difference in the final optimization results. (The error between $x_{t}$ and $\hat{x}_{t}$ cannot be larger than the saturated moisture content of the soil, so that the square of the saturated moisture content is the largest value any entry in $\mathbf{P}$ can "reasonably" be. This value was used for $\mathbf{P}_{0}^{+}$.) For our case the measurement noise variance (the $\mathbf{R}$ matrix) can be taken from the standard error of the neutron probe instrument, which was found in a separate calibration to be $2.1 \%$. We set the hydraulic conductivity parameters $A$ and $B$ in an optimization process.

In the Kalman filter approach, the estimation error $e_{i, t}$ is taken to have a Gaussian distribution with zero mean. An explicit likelihood function can be written for it, which after some manipulation can be expressed as [Shumway, 1988]

$$
\log L=\sum_{t=1}^{t=n}\left[-\frac{1}{2} \log \left|\mathbf{P}_{t}^{-}\right|-\frac{1}{2}\left(\boldsymbol{\theta}_{t}-\mathbf{H} \hat{\mathbf{x}}_{t}^{-}\right) \mathbf{P}_{t}^{-}\left(\boldsymbol{\theta}_{t}-\mathbf{H} \hat{\mathbf{x}}_{t}^{-}\right)^{T}\right]
$$

where $n$ is the number of values in the time series. Since the values of $\hat{\mathbf{x}}_{t}^{-}$and $\mathbf{P}_{t}^{-}$depend in a complex way on $A$ and $B$, the 


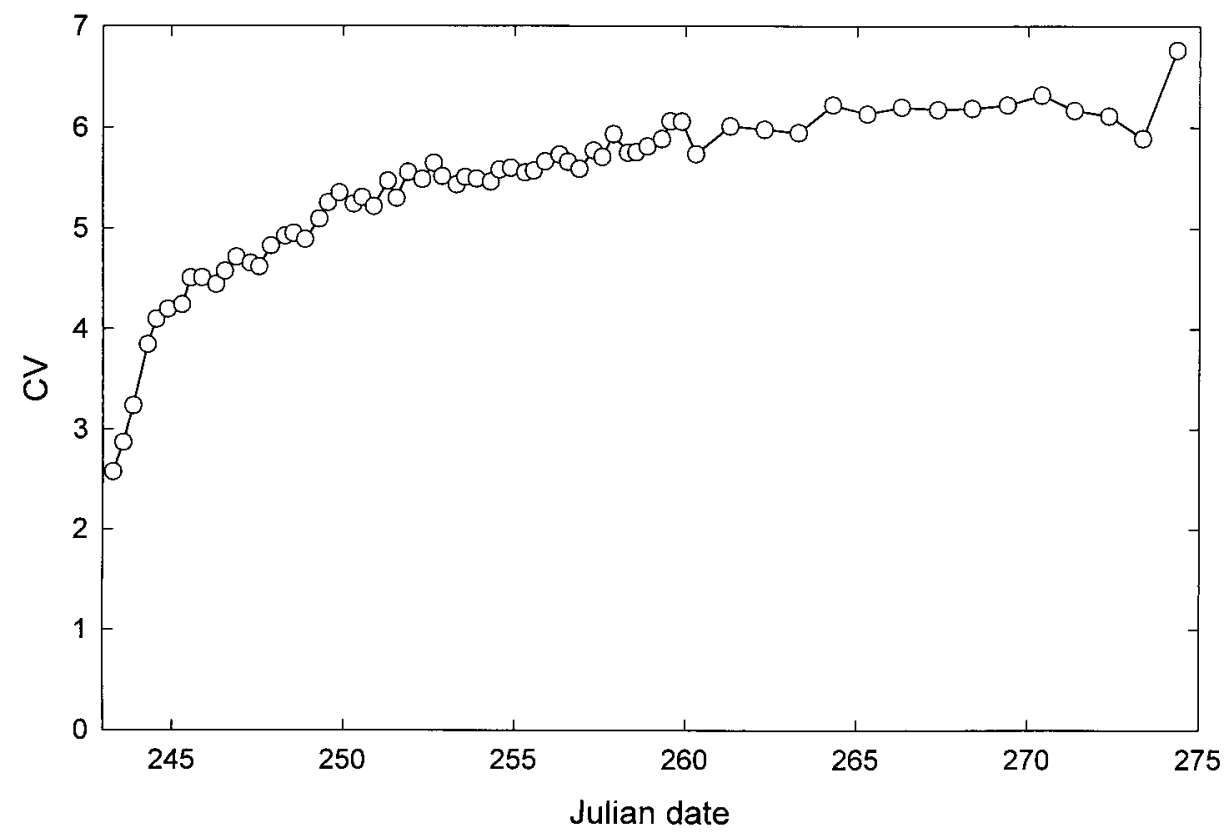

Figure 2. The coefficient of variation (mean divided by standard deviation) of the $\theta_{*}$ values at $65 \mathrm{~cm}$. It is apparent that as the soil dries, the measured soil moisture at the measurement locations becomes increasingly different.

value of $L$ is determined by these parameters. Conversely, the values of $A$ and $B$ which yield the maximum value for $L$ are the hydraulic conductivity parameters which give a time series for $\hat{\mathbf{x}}_{t}$ which is most like the unknown true time series of soil moisture content $\mathbf{x}_{t}$. Because of the nonlinearity in the time propagation equation and the likelihood function, we need to find $A$ and $B$ which yield the maximum value of $L$ by iteration. In practice, we add a fifth step to the four above:

Step 5. Calculate the likelihood $L$ using (12). Adjust the values of $A$ and $B$ to improve the likelihood, and return to step 1 , unless some convergence criterion for the value of the likelihood function is met.

Once the convergence criterion is met, $A$ and $B$ give our optimal estimates for the hydraulic conductivity function $K$. The Powell algorithm [Press et al., 1986] was used for determination of how to change $A$ and $B$ from iteration to iteration and for a convergence test for the likelihood function.

The final parameter needed, the $\mathbf{Q}$ matrix, is often the most difficult parameter of the Kalman filter algorithm to supply. It is possible to allow $\mathbf{Q}$ to be one of the parameters for which the Kalman filter is optimized. With $n$ different state variables, however, the $\mathbf{Q}$ matrix has $n^{2}$ elements. As $n^{2}$ approaches the number of time periods observed, the confidence with which any $\mathbf{Q}_{i j}$ can be estimated by an optimization method becomes small. It is useful therefore to have a model for $\mathbf{Q}$. For this we will turn to spatial statistics.

\subsection{Spatial Statistics}

Field spatial heterogeneity introduces the major uncertainty factor for field-scale water content estimates [Haverkamp et al., 1984; Vauclin et al., 1984; Parlange et al., 1992]. In the statespace formulation the stochastic noise component associated with the scale inherent spatial variability can be defined and modeled using semivariogram functions if measurements at different locations are available. We first describe the assump- tions made for the spatial analysis and some observations on the empirical spatial statistics.

In performing exploratory data analysis on the moisture content data, it was noticed that the amplitude of the variogram function, and hence the sill, steadily increased over the duration of the experiment, while the first lag semivariances were not significantly different from the mean value over the entire duration of the experiment accordingly to the $t$ test of significancy (significance level, $p=0.05$ ). This behavior can be explained by observing that the coefficient of variation (standard deviation of the measurements of each day, or daily standard deviation, divided by the mean of each day) and the variance of the soil water content (averaged over the 12 locations) data set increased over time too (see Figure 2), affecting the amplitude but not the shape of the experimental variographies. A steadily increasing coefficient of variation was observed by Greminger et al. [1985] during a similar experiment on the same soil, but in that case the daily standard deviation was nearly invariant.

A simple approach to this problem is to assume that the shape of the variogram is the same over the entire duration of the experiment but that its amplitude changes from day to day [Isaaks and Shrivastava, 1989]. This simplifying assumption allows us to use a single variogram model, $\gamma_{R}(h)$, rescaling the daily experimental variograms to the daily sills. It is important to notice that rescaling a variogram to some constant does not change its shape. Therefore the estimates will not be modified. While the kriging estimates are not affected by rescaling the variogram, its amplitude affects the estimation variance. If this relative model is used in a test kriging procedure to calculate the kriging variance as an index of uncertainty of the spatial model, the result is an error variance, $\sigma_{R}^{2}$, which is relative to the daily variance. In order to assess the actual variance, $\sigma_{t}^{2}$, this relative value must be rescaled to some estimate of the daily variance, $\sigma_{* \text {,daily: }}^{2}$ : 
Table 1. Regression Parameters Daily Soil Water Content Moving Average Means Versus Daily Standard Deviations

\begin{tabular}{rrrl}
\hline Site & $\begin{array}{c}\text { Correlation } \\
\text { Coefficient }\end{array}$ & Intercept & Slope \\
\hline 1 & 0.811 & -0.05268 & 0.281 \\
2 & 0.865 & -0.04364 & 0.237 \\
3 & 0.897 & -0.05385 & 0.28 \\
4 & 0.848 & -0.05273 & 0.259 \\
5 & 0.806 & -0.05984 & 0.275 \\
6 & 0.844 & -0.06029 & 0.278 \\
7 & 0.874 & -0.06633 & 0.312 \\
8 & 0.844 & -0.06251 & 0.303 \\
9 & 0.864 & -0.05885 & 0.293 \\
10 & 0.801 & -0.04698 & 0.237 \\
11 & 0.860 & -0.04647 & 0.246 \\
12 & 0.716 & -0.04519 & 0.239 \\
\hline
\end{tabular}

$\sigma_{t}^{2}=\sigma_{* \text {,daily }}^{2}\left[2 \sum_{i=1}^{N-1} \lambda_{i} \gamma_{R}\left(\mathbf{x}_{0}, x_{i}\right)-\sum_{j=1}^{N-1} \sum_{i=1}^{N-1} \lambda_{i} \lambda_{j} \gamma_{R}\left(x_{i}, x_{j}\right)\right]$

where $\gamma_{R}$ is the relative semivariance and $\lambda_{i}$ is the kriging weights. The variance for each day (daily variance) is usually related to the daily mean. A scatter plot of moving average daily means versus daily variances for each location reveals the existence of such a relationship (proportional effect) and eventually provide a linear regression equation to predict daily variance from the daily mean. The correlation coefficients computed at each location are rather large, ranging from 0.716 (location 12) to 0.897 (location 3), and the results for the 12 locations are shown in Table 1 . The kriged daily estimates for the soil moisture content at each location (using the absolute variogram model) will then provide the mean values necessary

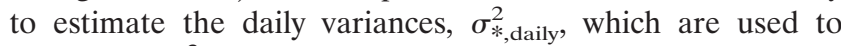
rescale the $\sigma_{R}^{2}$ calculated with the relative semivariogram.

\subsection{Process Noise Covariance Model}

In the context of this paper semivariograms were not used for spatial prediction but for estimating the $\mathbf{Q}$ matrix as one of the parameters for Kalman filter optimization. The modeled semivariograms are used to scale the empirical daily variance of the measurements $\boldsymbol{\theta}$ to yield estimates for the entries of the Q matrix. The state-space equations (2) and (4) are used to derive a relationship between the transition process noise $w_{i, t}$ and the observations $\theta_{i, t}$, the observation noise (both of which are known), and the modeled transition process (equation (5)). This allows the estimation of the entries of the $\mathbf{Q}$ matrix, which are by definition $E\left(w_{i, t} w_{j, t}\right)$, where $E(\quad)$ is the expectation operator.

Noting that $\mathbf{H}$ is a unit vector which can be ignored, we use (2) and (4) to write

$$
\begin{aligned}
w_{i, t}= & \theta_{i, t+1}-v_{i, t+1}-\theta_{i, t}+v_{i, t} \\
& -\int_{t}^{t+1}-A \exp \left(B\left(\theta_{i, t}-v_{i, t}\right)\right) \frac{\partial h}{\partial z} d \hat{t}
\end{aligned}
$$

which for notation convenience, we will shorten to

$w_{i, t}=\theta_{i, t+1}-v_{i, t+1}-\theta_{i, t}+v_{i, t}-\int_{t}^{t+1} f\left(\theta_{i, t}-v_{i, t}\right) d \hat{t}$

The entries in the $\mathbf{Q}$ matrix can then be written

$$
\begin{aligned}
Q_{i j}= & E\left(w_{i, t} w_{j, t}\right) \\
= & E\left\{\left(z_{i, t+1}-v_{i, t+1}-z_{i, t}+v_{i, t}-\int_{t}^{t+1} f\left(z_{i, t}+v\right) d \hat{t}\right)\right. \\
& \left.\cdot\left(z_{j, t+1}-v_{j, t+1}-z_{j, t}+v_{j, t}-\int_{t}^{t+1} f\left(z_{j, t}+v\right) d \hat{t}\right)\right\} \quad(16) \\
= & \operatorname{Cov}\left[z_{i, t+1} z_{j, t+1}+z_{i, t} z_{j, t}-z_{i, t+1} z_{j, t}-z_{j, t+1} z_{i, t}\right] \\
& +E\left\{-z_{i, t+1} \int_{t}^{t+1} f\left(z_{j, t}+v\right) d \hat{t}+z_{i, t} \int_{t}^{t+1} f\left(z_{j, t}+v\right) d \hat{t}\right. \\
& -z_{j, t+1} \int_{t}^{t+1} f\left(z_{i, t}+v\right) d \hat{t}+z_{j, t} \int_{t}^{t+1} f\left(z_{i, t}+v\right) d \hat{t} \\
& \left.+\int_{t}^{t+1} f\left(z_{i, t}+v\right) d \hat{t} \int_{t}^{t+1} f\left(z_{j, t}+v\right) d \hat{t}\right\}+2 R
\end{aligned}
$$

where Cov indicates covariance and $R$ is the time-independent covariance matrix of $v$.

We now make simplifying assumptions to allow numerical determination of $Q_{i j}$. For $f\left(z_{j, t}+v\right)=A \exp \left(B\left(z_{j, t}+\right.\right.$ $v))\left(\partial h_{i} / \partial z\right)$, because of the independence of the measurement error from the measurement value we can write

$$
\begin{aligned}
E[A & \left.\exp \left(B\left(z_{i, t}+v\right)\right) \frac{\partial h_{i}}{\partial z}\right] \\
= & A \frac{\partial h_{i}}{\partial z} E\left[\exp \left(B z_{i, t}\right)\right] E[\exp (B v)]
\end{aligned}
$$

and approximating for $v$ small relative to the measurements (i.e., a Taylor series expansion is valid)

$$
\begin{aligned}
E[\exp (B v)] & =\exp (B \cdot E[v]) \\
& =\exp (B \cdot 0)=1
\end{aligned}
$$

The fact that there are multiplicative terms of $z_{j, t+1}$ and $z_{j, t}$ with $E[\exp (B v)]$ should not change the validity of this approximation, since the expectation of their product can be separated by the same independence argument. We will also expand the remaining $\exp \left(B z_{i, t}\right)$ in a power series and neglect higher-order terms (since $z$ is always less than one) and approximate the integral

$$
\begin{aligned}
& \operatorname{Cov}\left(w_{i, t} w_{j, t}\right) \\
& \approx\left(1-A B \Delta t \frac{\partial h_{i}}{\partial t}-A B \Delta t \frac{\partial h_{j}}{\partial t}+(A B \Delta t)^{2} \frac{\partial h_{i}}{\partial t} \frac{\partial h_{j}}{\partial t}\right) \\
& \quad \cdot \operatorname{Cov}\left[z_{i, t+1} z_{j, t+1}-z_{i, t+1} z_{j, t}-z_{j, t+1} z_{i, t}+z_{i, t} z_{j, t}\right]
\end{aligned}
$$

We now scale all $z$ terms by their respective daily standard deviations so that the stationary model given from the spatial analysis can be used to represent the covariances:

$$
\begin{aligned}
\operatorname{Cov}[ & \left.z_{i, t+1} z_{j, t+1}-z_{i, t+1} z_{j, t}-z_{j, t+1} z_{i, t}+z_{i, t} z_{j, t}\right]=\sigma_{t+1}^{2} C_{i j} \\
& -2 \sigma_{t+1} \sigma_{t} C_{i j}+\sigma_{t}^{2} C_{i j}
\end{aligned}
$$




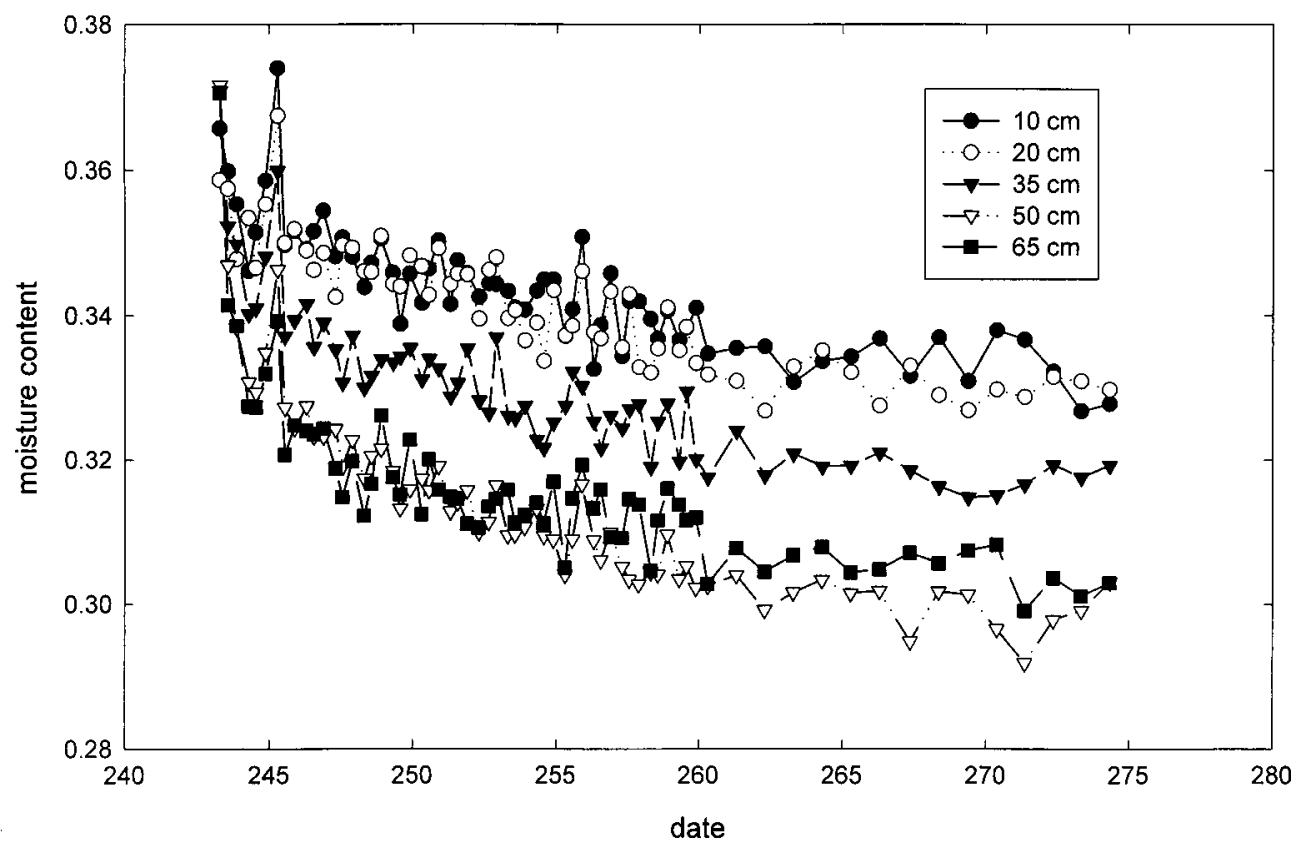

Figure 3. The volumetric soil moisture contents measured by neutron probe at the noted depths. The measurements are from column 7.

where the $\sigma$ s are given from $\bar{s}$, the measurement standard deviation, and the $C_{i j}$ are given by the spatial continuity model. The entries of the $\mathbf{Q}$ matrix are then

$$
\begin{aligned}
\mathbf{Q}_{i j}= & \left(1-A B \Delta t \frac{\partial h_{i}}{\partial t}-A B \Delta t \frac{\partial h_{j}}{\partial t}+(A B \Delta t)^{2} \frac{\partial h_{i}}{\partial t} \frac{\partial h_{j}}{\partial t}\right) \\
& \cdot\left(\sigma_{t+1}^{2}-2 \sigma_{t+1} \sigma_{t}+\sigma_{t}^{2}\right) C_{i j}+2 R
\end{aligned}
$$

This procedure will tend to underestimate the $\mathbf{Q}$ matrix, because of the approximation in linearizing the integral of $f\left(z_{i, t}\right.$ $+v)$. If the underestimation of the $\mathbf{Q}$ matrix leads to control problems (i.e., divergence of the estimated moisture content from its true value), the matrix entries can be increased by including higher-order terms in the series expansion of $f$. In light of the small size of higher powers of $z$ and the concessions to suboptimality inherent in the extended Kalman filter formulation, the effort required to develop a model for the spatial continuity of third-order spatial moments does not seem necessary.

\section{Results and Discussion}

Although a dozen measurement stations were installed, only six yielded sufficient continuous data for use in the analysis. Experimental problems included water seeping into one neutron probe access tube and a few unreliable tensiometers. There was no bias toward either end of the transect in which six stations were finally used. The measured moisture contents at the given depths for one of the access tubes are shown in Figure 3. It can be seen that from the plot of the hydraulic gradients that the assumption of a unit hydraulic gradient is true only on the average and that a model that accounted for this variation, whether because of measurement noise or warming of water in the tensiometer, might be more realistic. (This suggestion was not tried in this study because of the lack of information about the measurement noise characteristic of the pressure transducer and the effect of temperature on the tensiometers used.)

It should be noted that the method described in this paper is an inverse method: it derives a functional relationship on the basis of observation and as such shares the shortcoming of any inverse method. The extension of the hydraulic conductivity relationship beyond the range of moisture contents observed is improper and may yield wrong answers. However, if the drainage process is accurately modeled as Darcian flow, the hydraulic conductivity relationship derived should be applicable for any other flow process in the soil, including infiltration, if the moisture content range is similar.

To develop the variogram model, a Gaussian variogram model was fitted to the second day's data; this model is often used to model extremely continuous phenomena [Isaaks and Srivastava, 1989]:

$\gamma(h)=2.59 \times 10^{-5}+7 \times 10^{-5} \times\left[1-\exp \left(\frac{-3|h|^{2}}{4.1398^{2}}\right)\right]$

Then the relative semivariogram, jointly modeled on days 2,9 , 17 , and 32 data sets after standardization with their sill values is derived:

$\gamma(h)=0.02993 \overline{3}+0.97993 \overline{3} \times\left[1-\exp \left(\frac{-3|h|^{2}}{4.1398^{2}}\right)\right]$

Three of these days $(2,17$, and 32) were chosen to cover the full time range of the experiment; the fourth day (9) was chosen because there appeared to be greater variability in the first half of the experiment.

This scaled semivariogram (Figure 4) describes the spatial structure of the average soil water content at different times, taking into account the presence of the proportional effect over time. According to the selected model, the range of spatial autocorrelation is of about $4 \mathrm{~m}$, while for the same soil (Yolo clay loam, fine silty, mixed, nonacid, thermic, Typic 


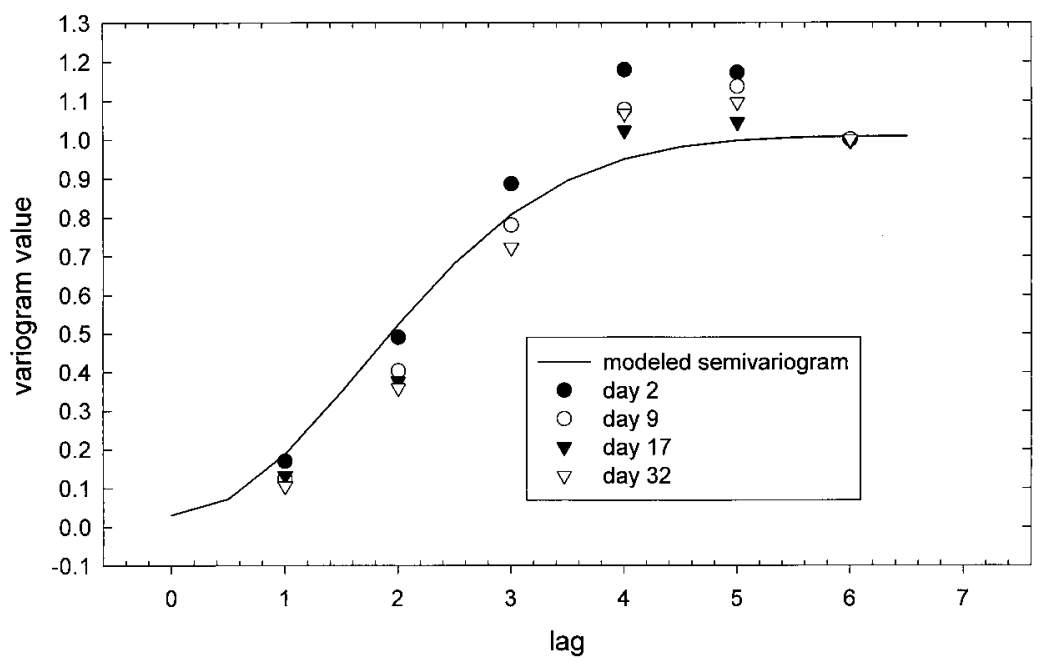

Figure 4. The model scaled semivariogram versus lag distance (meters). This model semivariogram value is multiplied by the variance of the time period for use in the computation of the $\mathbf{Q}$ matrix.

Xerorthents), sampled $1 \mathrm{~m}$ apart along a $100 \mathrm{~m}$ transect, Greminger et al. [1985] reported that values of soil water content measured at $60 \mathrm{~cm}$ depth were correlated no more than 1 or $2 \mathrm{~m}$. This could be due to the shorter transect sampled in our experiment and to the fact that the soil water content was averaged over a $65 \mathrm{~cm}$ depth, so that the measured values reflected the behavior of drainage properties of the entire soil profile rather than that of the subsoil alone. Additionally, the thorough soaking applied to the field at the beginning homogenized the soil moisture content, increasing the correlation length. These findings agree with results from previous experiments, considering that the number of significant lags of spatial dependence for the topsoil alone $(30 \mathrm{~cm})$ observed by Greminger et al. [1985] ranged from 6 to 7 over the length of the experiment.

In order to assess the robustness and the performance of the model, the variogram was validated with a test-kriging procedure, discarding one observation at a time and estimating it from the remaining samples using the fitted variogram model; the procedure was repeated for all the daily data sets. This led to a daily mean estimation error of $-0.000365\left(\mathrm{~cm}^{3} / \mathrm{cm}^{3}\right)$, a daily mean kriging variance of $0.00048\left(\mathrm{~cm}^{3} / \mathrm{cm}^{3}\right)$ and an average correlation coefficient between observed and estimated soil water content at each site of 0.918 .

The mean daily estimation error, submitted to the $t$ test for significance, was not significantly different from zero at the significance level $p=0.01$, verifying the unbiasedness condition of the kriging estimation and the daily estimation error had $100 \%$ of its values within the interval $\varepsilon \pm 2 \sigma \varepsilon$, where $\varepsilon$ is the daily observed mean error and $\sigma$ its standard deviation. Furthermore the daily point correlation coefficients were not significantly different from 1.0 at the 0.05 significance level. Those results ensure the goodness of the variogram model selected for characterizing the spatial structure of soil water content under the condition imposed in this experiment, despite the small number of observations available in the space domain. Furthermore those results should be judged considering that the error associated with geometric and instrument consideration of the neutron probes measurements is 0.01365 $\left(\mathrm{cm}^{3} / \mathrm{cm}^{3}\right)$.

For comparison, three other time series methods for determining the unsaturated hydraulic conductivity were applied. The theta, flux, and Chong et al. [1981] CGA methods [Libardi et al., 1980] all use the assumption of a unit hydraulic gradient to simplify (1), so that simple linear regressions can yield values for the parameters in the hydraulic conductivity equation. These methods use the measurements from a single drainage column, so that six sets of hydraulic conductivity parameters can be derived by each method.

The optimized values for $A$ and $B$ found by the Kalman filter method are given in Table 2, along with the standard deviations in the parameters given by the Fisher information criterion. The Kalman-filtered time series calculated using these optimal parameters are shown in Figure 5, along with the measured values of $\theta_{*}$. It can be seen that the Kalman filter using the optimal coefficients does a good job of tracking and estimating the moisture content measurements, except for time periods when there is abnormally high noise in the mea-

Table 2. Hydraulic Conductivity Function Parameters From Different Models

\begin{tabular}{lllll} 
& & & \multicolumn{2}{c}{ Standard Deviations } \\
\cline { 4 - 5 } Model & \multicolumn{1}{c}{$A$} & \multicolumn{1}{c}{$B$} & \multicolumn{1}{c}{$A$} & \multicolumn{1}{c}{$B$} \\
\hline Kalman & $5.72 \mathrm{E}-13$ & 95.5671 & $3.34 \mathrm{E}-14$ & 1.124016304 \\
Theta & 2.330994876 & 98.23754703 & 0.868862362 & 17.72524298 \\
Flux & 3.344817814 & 60.78484408 & 1.742240107 & 12.16375697 \\
CGA & 2.2640439 & 92.64128661 & 0.810614111 & 18.3894249 \\
\hline
\end{tabular}

The hydraulic conductivity for the Kalman filter model is given by $K=A \exp \left(B \theta^{*}\right)$; for the other three models it is $K=A \exp \left(B\left(\theta^{*}-\theta_{0}^{*}\right)\right)$. 

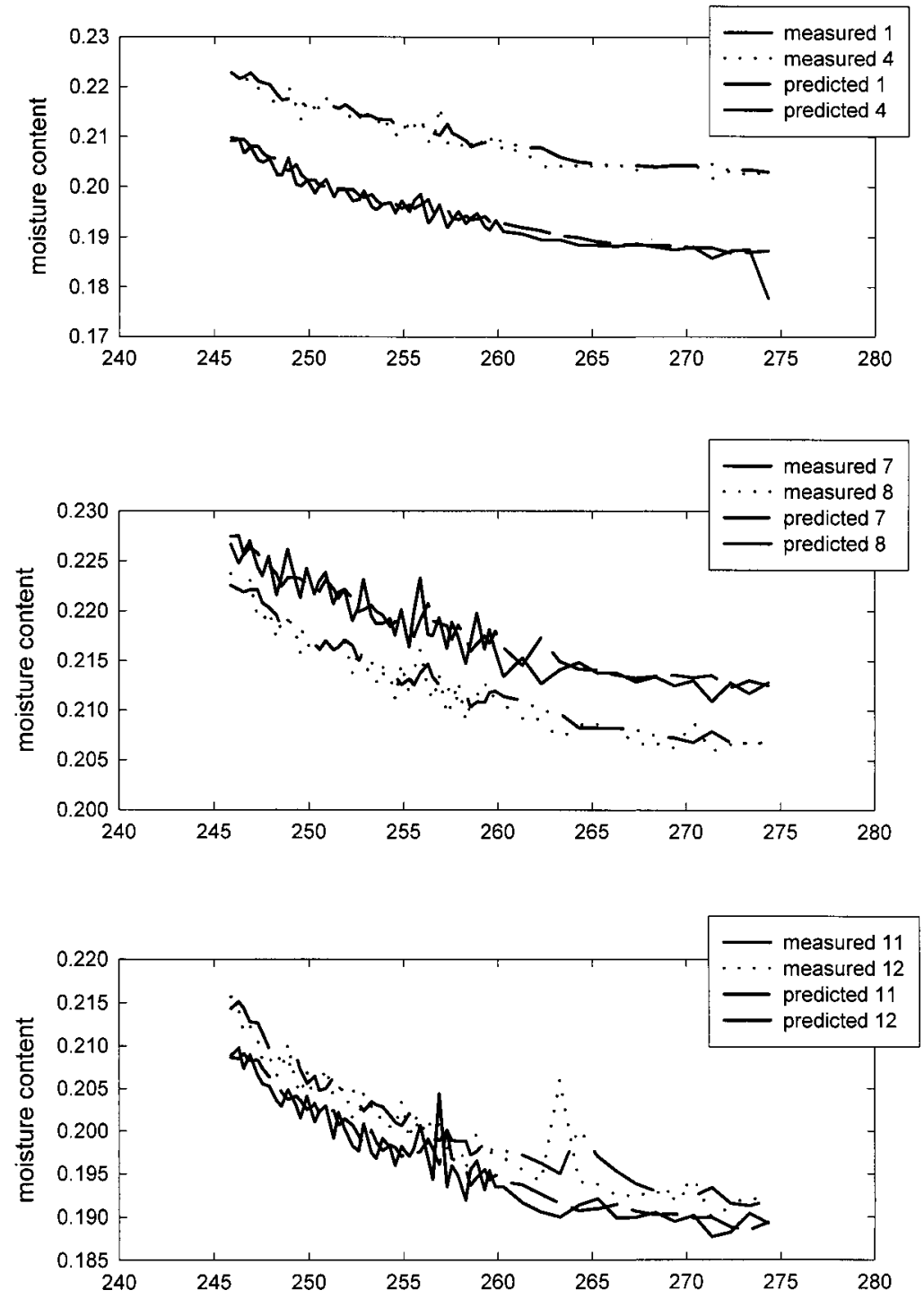

Figure 5. The measured soil moisture content and the soil moisture content estimated by the Kalman filter model.

surement, such as occurs around day 263 for station 12 (see Figure 5c). Table 2 also contains the average values of the parameters given by the three other time series analysis methods, along with the sample standard deviations. The hydraulic conductivity functions resulting from the parameters in Table 2 are shown in Figure 6. As can be seen, the uncertainty in the Kalman filter-derived hydraulic conductivity is much less than the uncertainty in any of the curves derived by the other methods. It is interesting to note that the Kalman filter result falls within the standard deviations of both the theta and CGA methods, while the results from the flux method are well out of range of the others. The results from this study are also consistent with those found by Katul et al. [1993], who considered only one spatial location.

\section{Conclusions}

The inverse methodology utilizing the Kalman filter presented in this study offers a means of deriving parameters for effective hydraulic conductivity equations with greater precision than simple averaging of the point parameters. The parameters from the Kalman filter method yielded a hydraulic conductivity function that was within one standard deviation of two of the less precise averaged methods, indicating that while simple averaging approximates reasonably well the optimal parameters, the spatial correlations among the sampling stations contain important information. The fact that the modulus of the Kalman gain matrix was approximately 0.5 suggests that the magnitude of the transition noise $w_{t}$ in this model is of the same order as the measurement noise. Some of the transition noise certainly arises from the neglect of measurement noise in the hydraulic gradient measurements and could be reduced by more accurate measurements of both moisture content and matric potential. The relatively small standard deviations of the parameters derived by the Kalman filter method compared to the averaged parameters of the other methods may in part be due to the different objectives: the other time series meth- 


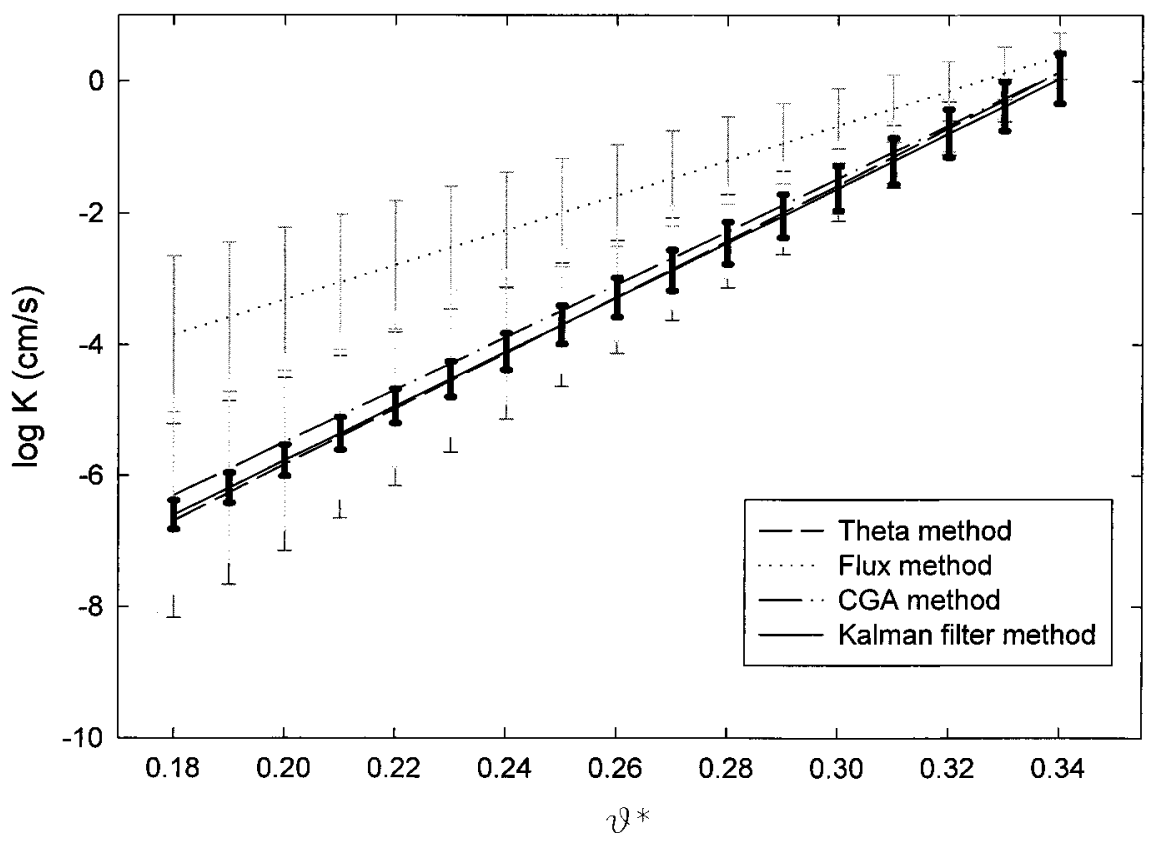

Figure 6. The hydraulic conductivity functions derived by the different methods versus $\theta_{*}$, the depthaveraged soil moisture content, along with error bars. The height of the solid bars on the Kalman filterderived line indicates the size of the standard deviation at that soil moisture content.

ods attempt to find the best fit for each individual sampling point, while the Kalman filter method finds the parameters that give the best fit for all the samples considered together. Finally, it is difficult to compare the results given by our method to more complex spatial averaging schemes, such as that suggested by Mantoglou [1992], because of the small number of spatial sampling points. The method presented here takes advantage of the relatively large number of time samples compared to the number of spatial samples. Further field research is needed to allow additional comparison of different methods of parameter estimation and to explore in what sense spatial and temporal changes in hydraulic conductivity are ergodic.

Acknowledgments. The first author was supported in part by a NASA Earth Systems Sciences Fellowship during this research. The second author was supported by a fellowship under the OECD Cooperative Research Program "Biological Resource Management for Sustainable Agricultural Systems; 1995 Postdoctoral Fellowship Program, Theme 4." The authors would also like to thank Fernando Porté Age for assistance in the experiment and acknowledge USDA, 98-351076346.

\section{References}

Brown, R. G., and P. Y. C. Hwang, Introduction to Random Signals and Applied Kalman Filtering, 3rd ed., 484 pp., John Wiley, New York, 1997.

Chen, Z., R. S. Govindaraju, and M. L. Kavvas, Spatial averaging of unsaturated flow equations under infiltration conditions over areally heterogeneous fields, 1, Development of models, Water Resour. Res., 30, 523-533, 1994.

Chong, S. K., R. E. Green, and L. R. Ahuja, Simple in situ determination of hydraulic conductivity by power function descriptions of drainage, Water Resour. Res., 17, 1109-1114, 1981.

Gelb, A. (Ed.), Applied Optimal Estimation, 374 pp., MIT Press, Cambridge, Mass., 1974.

Greminger, P. J., Y. K. Sud, and D. R. Nielsen, Spatial variability of field-measured soil-water characteristics, Soil Sci. Soc. Am. J., 49, 1075-1082, 1985.

Haverkamp, R., M. Vauclin, and G. Vachaud, Error analysis in estimating soil water content from neutron probes measurements, 1 , Local standpoint, Soil Sci., 137, 78-90, 1984.

Katul, G. G., O. Wendroth, M. B. Parlange, C. E. Puente, M. V. Folegatti, and D. R. Nielsen, Estimation of in situ hydraulic conductivity function from nonlinear filtering theory, Water Resour. Res., 29, 1063-1070, 1993.

Isaaks, H., and R. M. Srivastava, An Introduction to Applied Geostatistics, 561 pp., Oxford Univ. Press, New York, 1989.

Libardi, P. L., K. Reichardt, D. R. Nielsen, and J. W. Biggar, Simple field methods for estimating soil hydraulic conductivity, Soil Sci. Soc. Am. J., 44, 3-7, 1980.

Mantoglou, A., A theoretical approach for modeling unsaturated flow in spatially variable soils: Effective flow models in finite domains and nonstationarity, Water Resour. Res., 28, 251-267, 1992.

Mantoglou, A., and L. W. Gelhar, Stochastic modeling of large-scale transient unsaturated flow systems, Water Resour. Res., 23, 37-46, 1987a.

Mantoglou, A., and L. W. Gelhar, Capillary tension head variance, mean soil moisture content, and effective specific soil moisture capacity in transient unsaturated flow in stratified soils, Water Resour. Res., 23, 47-56, 1987b.

Mantoglou, A., and L. W. Gelhar, Effective hydraulic conductivities of transient unsaturated flow in stratified soils, Water Resour. Res., 23, $57-67,1987 \mathrm{c}$.

Maybeck, P. S., Stochastic Models, Estimation and Control, vol. 2, 289 pp., Academic, San Diego, Calif., 1982.

Nielsen, D. R., J. W. Biggar, and K. T. Erh, Spatial variability of field-measured soil-water properties, Hilgardia, 42, 215-260, 1973.

Parlange, M. B., G. G. Katul, R. H. Cuence, M. L. Kavvas, D. R. Nielsen, and M. Mata, Physical basis for a time series model of soil water content, Water Resour. Res., 28, 2437-2446, 1992.

Parlange, M. B., G. G. Katul, M. V. Folegatti, and D. R. Nielsen, Evaporation and the field scale soil-water diffusivity function, Water Resour. Res., 29, 1279-1286, 1993.

Press, W. H., S. A. Teukolsky, W. T. Vetterling, and B. P. Flannery, Numerical Recipes in C, Cambridge Univ. Press, New York, 1992.

Quintard, M., and S. Whitaker, Two-phase flow in heterogeneous porous media: The method of large-scale averaging, Transp. Porous Media, 3, 357-413, 1988. 
Shumway, R. H., Applied Statistical Time Series Analysis, 380 pp., Prentice-Hall, Englewood Cliffs, N. J., 1988.

Tseng, P.-H., and W. A. Jury, Simulation of field measurements of hydraulic conductivity in unsaturated heterogenous soil, Water Resour. Res., 29, 2087-2099, 1993.

Vauclin, M., R. Haverkamp, and G. Vachaud, Error analysis in estimating soil water content from neutron probes measurements, 1 , Spatial standpoint, Soil Sci., 137, 141-148, 1984.

Viera, S. R., D. R. Nielsen, and J. W. Biggar, Spatial variability of field-measured infiltration rate, Soil Sci. Soc. Am. J., 45, 1040-1048, 1981.

Wendroth, O., G. G. Katul, M. B. Parlange, C. E. Puenter, and D. R. Nielsen, A nonlinear filtering approach for determining hydraulic conductivity functions in field soils, Soil Sci., 156, 293-301, 1993.

Wierenga, P. J., R. G. Hills, and D. B. Hudson, The Las Cruces trench site: Characterization, experimental results, and one-dimensional flow predictions, Water Resour. Res., 27, 2695-2705, 1991.

Yeh, T. C., L. W. Gelhar, and A. L. Gutjahr, Stochastic analysis for unsaturated flow in heterogeneous soil, 1 , Statistically isotropic media, Water Resour. Res., 21, 447-456, 1985a.

Yeh, T. C., L. W. Gelhar, and A. L. Gutjahr, Stochastic analysis for unsaturated flow in heterogeneous soil, 2, Statistically anisotropic media with variable $\alpha$, Water Resour. Res., 21, 457-464, 1985b.

A. T. Cahill, Department of Civil and Environmental Engineering, Texas A\&M University, College Station, TX 77843-3136.

M. Mata and D. R. Nielsen, Department of Land, Air and Water Resources, Veihmeyer Hall, University of California, Davis, CA 95616.

M. B. Parlange, Department of Geography and Environmental Engineering, Johns Hopkins University, Ames Hall, Baltimore, MD 21218. (mbparlange@jhu.edu)

F. Ungaro, Soil Genesis and Ecology Institute, CNR-IGES, Florence, Italy 50144.

(Received May 27, 1998; revised December 15, 1998; accepted December 15, 1998.) 